\title{
An Approach to Assessment of Female Urinary Incontinence Risk using the Thickness of the Transverse Abdominal Muscle during Co-contraction of both the Transverse Abdominal Muscle and the Pelvic Floor Muscle
}

\author{
Kimiko TAJiri ${ }^{1)}$, Ming $\mathrm{HuO}^{2)}$, Ke Yin $^{3)}$, Sigeko FuJisawa ${ }^{2)}$, Hitoshi MaruYAma ${ }^{2)}$ \\ 1) Department of Nursing, Faculty of Health Science, International University of Health and Welfare: \\ 2600-1 Kitakanemaru,Ohtawara City, Tochigi 324-8501 Japan. \\ TEL: +8128724 3000,E-mail: tajiri@iuhw.ac.jp \\ 2) Department of Physical Therapy, Faculty of Health Science, International University of Health and \\ Welfare \\ 3) Jilin Province Rehabilitation Center
}

\begin{abstract}
Purpose] This study examined physical factors associated with urinary incontinence (UI) in women. We hypothesized that, women with UI would show decreased thickness of the transverse abdominal muscle (TA) during maximal co-contraction of both TA and the pelvic floor muscle (PFM) compared with the women with no history of UI. [Subjects] The subjects were thirty-two women who subjects were divided into two groups: the UI group and the No-UI group. [Methods] We evaluated the thickness of TA by ultrasound, and hand-grip strength and the muscular strength of adduction in flexion of hip joint. The thickness of TA was measured during performance of 4 tasks: (1) at rest, (2) maximal contraction of TA, (3) maximal contraction of PFM, and (4) maximal co-contraction of both TA and PFM. [Results] The No-UI group had thicknesses of TA that were greater than those seen in the UI group in the tasks 3 and 4. In logistic regression analysis with UI as the dependent variable, the thickness of TA during maximal cocontraction was identified as an independent factor, and the cut-off value of the thickness of TA was $2.55 \mathrm{~mm}$ as determined by the Receiver-Operating-Characteristic (ROC) curve. [Conclusion] We found that the thickness of TA during maximal co-contraction of both TA and PFM is reliable and useful for the evaluating the risk of UI in women. Key words: Urinary incontinence, Transverse abdominal muscle, Pelvic floor muscle
\end{abstract}

(This article was submitted May 14, 2010, and was accepted Jun. 24, 2010)

\section{INTRODUCTION}

Urinary incontinence (UI) is well-known to profoundly affect women's QOL (quality of life). Three to five million people women are worried by UI beginning at the gravid period, the intrapartum period, the puerperal period, or the postmenopausal period.

Many cases of UI are stress urinary incontinence (SUI), and the success of pelvic floor muscle (PFM) exercise in the management of SUI has been confirmed by multiple randomized controlled studies. PFM exercise has been reported to be $50 \%$ to $69 \%$ effective at reducing urine loss episodes in women ${ }^{1-4)}$. In previous studies of female UI, we found that there are several ways of assessing severity of UI, such as bladder diary, pat test, and the urodynamic test. However, few studies have evaluated the risk of UI. Many UI cases are the result of
PFM weakness, suggesting that risk of UI can be evaluated by PFM. Recently, several studies reported that PFM as an inner unit with the transverse abdominal muscle (TA), the multifidus muscle, and the diaphragm acts to maintain the stability of the trunk, and PFM has begun to be used as an approach to not only UI but also lumbar pain ${ }^{5-7)}$. In our previous study, a significant relationship was found between the thickness of TA and the iEMG of the levator ani muscle. This suggests that changes in thickness of TA may be used to indicate changes in the electrical activity of PFM.

In this paper, we examined factors of physical function associated with UI. The hypothesis was that, women with UI would show decreased the thickness of TA during maximal co-contraction of both TA and PFM compared with the women with no history of UI. 


\section{SUBJECTS AND METHODS}

The subjects were thirty-two women who were divided into two groups: the UI group $(\mathrm{n}=7)$ and No-UI group $(n=25)$ (Table 1). All subjects were primiparous women. All subjects gave their informed consent to participation in the study.

We evaluated the thickness of TA by ultrasound, and hand-grip strength and muscle strength of adduction in flexion of hip joint.

To measure the thickness of TA, four tasks were randomly performed in the supine position. To examine the measurement reliability, measurements during the four tasks were repeated.

1. Resting state.

2. Maximal contraction of TA. Subjects were instructed to draw in the lower abdominal wall toward the spine, action which specifically activates TA. The subjects were required to breathe in a relaxed manner. No movement of the lumbar spine was allowed.

3. Maximal contraction of PFM. Subjects were instructed to contract the muscles around the vagina "like a drawstring" and to lift them internally. No posterior tilt of the pelvis was allowed. There was no instruction to either use or not use the abdominal muscles.

4. Maximal co-contraction of both TA and PFM.

Subjects performed this task in the supine position with the knees flexed at $90^{\circ}$, and with a pillow under the head. Ultrasound images of the antero-lateral abdominal wall were obtained using an SonoSite (SonoSite 180 PLUS, B mode, $5 \mathrm{MHz}$ linear transducer). Gel was interposed between the transducer and the skin and the transducer was positioned adjacent to and perpendicular to the abdominal wall $25 \mathrm{~mm}$ antero-medial to the midpoint between the ribs and ilium on the mid-axillary line and parallel to the muscle fibres of the transversus abdominis ${ }^{8)}$. The measurements and recordings were done by the same person, a midwife, to avoid inter-rater errors. Ultrasound images were saved as still images. All thickness measurements were of muscle only, that is, between the fascia boundaries.

To measure the muscular strength of adduction in
Table 1. Subject Characteristics ${ }^{\mathrm{a}}$

\begin{tabular}{lccr}
\hline & $\begin{array}{c}\text { No-UI } \\
(\mathrm{n}=25)\end{array}$ & $\begin{array}{c}\text { UI } \\
(\mathrm{n}=7)\end{array}$ & $\begin{array}{c}\text { Overall } \\
(\mathrm{n}=32)\end{array}$ \\
\hline Age $(\mathrm{y})$ & $45.8 \pm 5.4$ & $50.1 \pm 4.2$ & $46.8 \pm 5.4$ \\
Height $(\mathrm{cm})$ & $161.3 \pm 5.0$ & $154.9 \pm 7.5^{* *}$ & $159.9 \pm 6.2$ \\
Weight $(\mathrm{kg})$ & $59.0 \pm 6.3$ & $57.4 \pm 11.1$ & $58.7 \pm 7.5$ \\
\hline
\end{tabular}

Note: values are mean \pm standard deviation. $* *: p<0.01$, ${ }^{\mathrm{a}} \mathrm{UI}$ group: woman with urinary incontinence. No-UI group: woman with no history of urinary incontinence.

was used to measure the maximal muscular strength of adduction in flexion. Subjects were instructed to tighten the adductor muscles for 3 seconds as an isometric contraction. The test was performed twice, and the maximum value was used for the analysis as the representative value.

In order to determine the reliability of the measurement values of the thickness of TA, the interclass correlation coefficient (ICC) was calculated. To determine differences between the UI group and the NoUI group, the independent $t$-test was performed for each measure. To determine correlations between items, Pearson's correlation coefficient was used. Logistic regression analysis and the Receiver-OperatingCharacteristic (ROC) curve were used to investigate the accrual of urinary incontinence and its relation to each factor. The Hosmer \& Lemeshow test judged the adaptability of the logistic regression analysis. The data were analyzed using SPSS Ver. 12.0 for Windows.

\section{RESULTS}

Table 2 shows the values of the test-retest coefficients (ICC). The ICCs of the thickness of TA ranged from 0.88 to 0.96 for all tasks, showing high reproducibility $(\mathrm{p}<0.01)$.

The UI group had significantly decreased thicknesses of TA during maximal contraction of PFM $(\mathrm{p}<0.05)$ and maximal co-contraction of both TA and PFM $(\mathrm{p}<0.01)$ compared to the No-UI group. The hand-grip strength and the muscular strength of adduction in flexion showed

Table 2. Measurements and $\operatorname{ICC}^{\mathrm{a}}(1,1)$ of Thickness of $\mathrm{TA}^{\mathrm{b}}(\mathrm{mm})$ $(\mathrm{n}=32)$

\begin{tabular}{lccc}
\hline & First measurement & Second measurement & ICC $(1,1)$ \\
\hline Resting state & $2.3 \pm 0.4$ & $2.2 \pm 0.4$ & $0.95^{* *}$ \\
Maximal contraction of TA $^{\text {Maximal contraction of PFM }}{ }^{\mathrm{c}}$ & $2.4 \pm 0.4$ & $2.4 \pm 0.4$ & $0.87^{* *}$ \\
Maximal co-contraction $^{\text {d }}$ & $2.4 \pm 0.4$ & $2.4 \pm 0.5$ & $0.93^{* *}$ \\
\hline
\end{tabular}

Note: values are mean \pm standard deviation. $*: p<0.05, * *: p<0.01{ }^{a}$ ICC: interclass correlation coefficient.

${ }^{b}$ TA: transverse abdominal muscle. ${ }^{\mathrm{c}}$ PFM: pelvic floor muscle. ${ }^{\mathrm{d}}$ Maximal co-contraction: Maximal cocontraction both TA and PFM.

flexion, subjects were asked to sit on a chair. A hand-held dynamometer (HDD, ANIMA MT-1) was fixed to the medial side of both knees, and the tester function of HDD no significant differences between the UI group and the No-UI group (Table 3).

Table 4 shows the correlations among the items. 
There was a high correlation among the thickness of TA during maximal contraction of TA, maximal contraction of PFM, and maximal co-contraction.

Logistic regression analysis with urinary incontinences as the dependent variable of the hand-grip strength, the muscular strength of adduction in flexion, and the thickness of TA during maximal co-contraction of both TA and PFM was performed by the stepwise method. Only the thickness of TA during maximal cocontraction of both TA and PFM showed a significant result $(\mathrm{p}<0.05)$ (Table 5). For urinary incontinence as a variable of state, the ROC curve of the thickness of TA during maximal co-contraction of both TA and PFM was plotted (Fig. 1). The area under the curve (AUC) was $81 \%$ and the cut-off value was $2.55 \mathrm{~mm}$. The sensitivity was $100 \%$ and the specificity was $52 \%$ according to the cross-table of the cut-off value (Table 6).

\section{DISCUSSION}

The ICC of the thickness of TA was high, and showed high reproducibility. The purpose of this study was to examine physical factors associated with urinary incontinence in women. Supporting our hypothesis, the

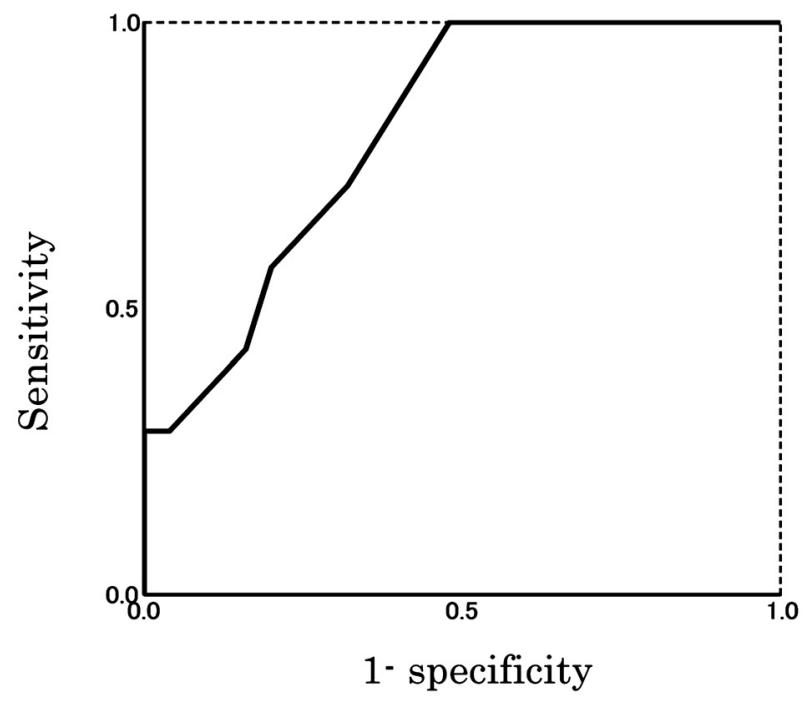

Fig. 1. The Receiver-Operating-Characteristic.

(ROC) curve of the thickness of TA during maximal cocontraction of both TA and PFM. The area under the curve (AUC) was $81 \%$, and the cut-off value was $2.55 \mathrm{~mm}$; the sensitivity was $100 \%$ and the specificity was $52 \%$. (Asymptotic significance probability $=0.014$ )

Table 3. Results of physical tests performed by the UI group and the No-UI group

\begin{tabular}{lcc}
\hline & No-UI group $(\mathrm{n}=25)$ & UI group $(\mathrm{n}=7)$ \\
\hline Hand-grip strength $(\mathrm{kg})$ & $25.5 \pm 6.2$ & $26.9 \pm 3.5$ \\
Muscular strength of adduction in flexion of hip joint $(\mathrm{kg})$ & $13.7 \pm 4.6$ & $14.6 \pm 3.8$ \\
Thickness of TA $^{\mathrm{a}}(\mathrm{mm})$ & & \\
Resting state $^{\text {Maximal contraction of TA }}$ & $2.4 \pm 0.4$ & $2.1 \pm 0.3$ \\
Maximal contraction of PFM $^{\mathrm{b}}$ & $2.5 \pm 0.4$ & $2.2 \pm 0.4$ \\
Maximal co-contraction $^{\mathrm{c}}$ & $2.5 \pm 0.4$ & $2.1 \pm 0.3^{*}$ \\
\hline
\end{tabular}

Mean $\pm \mathrm{SD},{ }^{*} \mathrm{p}<0.05,{ }^{* *} \mathrm{p}<0.01 .{ }^{\mathrm{a}} \mathrm{TA}$ : transverse abdominal muscle. ${ }^{\mathrm{b}} \mathrm{PFM}$ : pelvic floor muscle. ${ }^{\mathrm{c}}$ Maximal co-contraction: Maximal co-contraction both TA and PFM.

Table 4. Pearson Correlation Coefficients between Measures

\begin{tabular}{lcccccl}
\hline Hand-grip strength $^{\text {Adduction in flexion }}{ }^{\mathrm{a}}$ & - & $0.35^{*}$ & -0.13 & -0.18 & -0.11 & 0.10 \\
Resting state $^{-}$ & & 0.13 & 0.18 & 0.10 & 0.13 \\
Max. TA $^{\mathrm{b}}$ & & & - & $0.79^{* *}$ & $0.76^{* *}$ & $0.71^{* *}$ \\
Max. PFM $^{\mathrm{c}}$ & & & & - & $0.84^{* *}$ & $0.82^{* *}$ \\
Max. TA and PFM $^{\mathrm{d}}$ & & & & & - & $0.86^{* *}$ \\
\hline
\end{tabular}

*: $\mathrm{p}<0.05,{ }^{* *}: \mathrm{p}<0.01 .{ }^{\mathrm{a}}$ Adduction in flexion: Muscular strength of adduction in flexion of hip joint. ${ }^{b}$ Max. TA: Thickness of TA during maximal contraction of TA. ${ }^{\mathrm{c}}$ Max. PFM: Thickness of TA during maximal contraction of PFM. ${ }^{\mathrm{d}}$ Max. TA and PFM: Thickness of TA during maximal co-contraction both TA and PFM.

Table 5. Result of Logistic Regression Analysis with UI as the Dependent Variable

\begin{tabular}{lccc}
\hline Item & Odds Ratio & $95 \% \mathrm{CI}^{\mathrm{b}}$ & $\mathrm{p}$ \\
\hline Maximal co-contraction $^{\mathrm{a}}$ & 0.018 & $0.001-0.697$ & 0.03 \\
The Hosmer- Lemeshow Test $^{2}$ & $\chi^{2}=2.59$ & $\mathrm{p}=0.96$ & \\
\hline
\end{tabular}

Note: Stepwise way. ${ }^{a}$ Maximal co-contraction: Thickness of TA of maximal co-contraction 
Table 6. The cross-table of the cut-off value of the thickness of TA during maximal co-contraction of both TA and PFM

\begin{tabular}{lccc}
\hline & UI*group & No-UI group & Sum total \\
\hline$<2.55 \mathrm{~mm}$ & 7 & 12 & 19 \\
$\geq 2.55 \mathrm{~mm}$ & 0 & 13 & 13 \\
\hline Sum total & 7 & 25 & 32 \\
\hline
\end{tabular}

* UI: urinary incontinence. The sensitivity $=7 / 7=1.00$.

The specificity $=13 / 25=0.52$.

The positive predictive value $=7 / 19=0.37$.

The negative predictive value $=13 / 13=1.00$.

UI group had significantly decreased thicknesses of TA during maximal contraction of PFM and maximal cocontraction of both TA and PFM compared to the No-UI group. The hand-grip strength and the muscular strength of adduction in flexion showed no change between groups.

In logistic regression analysis, the thickness of TA of maximal co-contraction of both TA and PFM was identified as significant, indicating that the thickness of TA is useful for the evaluation of the risk of UI. In this research, the cut-off value of the thickness of TA during maximal co-contraction both TA and PFM was $2.55 \mathrm{~mm}$ according to the ROC curve, and the sensitivity was $100 \%$ and the specificity was $52 \%$. The result shows that the detectability of the risk of urinary incontinence was high, and quantitative assessment of the risk of UI is possible with measurement of the thickness of TA during maximal co-contraction of both TA and PFM. In addition, the negative predictive value was 100 percent and the predictive accuracy was 74 percent, greatly exceeding the percentage of women with UI, 21.9 percent. This demonstrates that a high-precision UI forecast is possible using this model. In conclusion, the thickness of TA during maximal co-contraction of both TA and PFM is useful for the evaluation of the risk of female urinary incontinence.

\section{REFERENCES}

1) Henalla SM, Hutchins CJ, Robinson P, et al.: Non-operative methods in the treatment of female genuine stress incontinence of urine. J Ob Gyn, 1989, 9: $222-225$.

2) Goode P, Burgio KL, Locher JL, et al.: Effect of behavioral training with or without pelvic floor electrical stimulation on stress incontinence in women: a randomized controlled trial. JAMA, 2003, 290: 345-352.

3) Hay-Smith EJC, Dumoulin C: Pelvic floor muscle training versus no treatment, or inactive control treatments for urinary incontinence in women for urinary incontinence. Cochrane Database Syst Rev, 2007, 2.

4) Borello-France DF, Zyczynski HM, Downey PA, et al.: Effect of pelvicfloor muscle exercise position on continence and quality-of-life outcomes in women with stress urinary incontinence. Phys Ther, 2006, 86: 974-986.

5) Hodges PW, Richardson CA: Contraction of the abdominal muscles associated with movement of the lower limb. Phys Ther, 1997, 77: 132-144.

6) Neumann P, Gill V: Pelvic floor and abdominal muscle interaction: EMG activity and intra- abdominal pressure. Int Urogynecol J Plevic Floor Dysfunct, 2002, 13: 125-132.

7) Carriere B: Fitness for the Pelvic Floor. Thieme Stuttgart, 2002, 14-37.

8) Critchiey DJ: Instructing pelvic floor contraction facilitates transversus abdominis contraction during low abdominal hollowing. Physiother Res Int, 2002, 7: 66-75. 